\title{
Metabolic syndrome and abdominal fat are associated with inflammation, but not with clinical outcomes, in peritoneal dialysis patients
}

Jenq-Wen Huang ${ }^{1}$, Chung-Yi Yang ${ }^{2}$, Hon-Yen Wu ${ }^{1,4}$, Kao-Lang Liư ${ }^{2}$ Chi-Ting Su' ${ }^{5}$ Cho-Kai Wu' ${ }^{1}$, Jen-Kuang Lee ${ }^{8}$, Chih-Kang Chiang ${ }^{1,3}$, Hui-Teng Cheng ${ }^{6}$, Yu-Chung Lien ${ }^{7^{*}}$ and Kuan-Yu Hung ${ }^{1^{*}}$

\begin{abstract}
Background: In the general population, metabolic syndrome (MetS) is correlated with visceral fat and a risk factor for cardiovascular disease (CVD); however, little is known about the significance of abdominal fat and its association with inflammation and medication use in peritoneal dialysis (PD) patients. We investigated the relationship of visceral fat area (VFA) with C-reactive protein (CRP) levels and medication use in PD patients and followed their clinical outcomes.
\end{abstract}

Methods: In a prospective study from February 2009 to February 2012, we assessed diabetes mellitus (DM) status, clinical and PD-associated characteristics, medication use, CRP levels, components of MetS, and VFA in 183 PD patients. These patients were categorized into 3 groups based on MetS and DM status: non-MetS (group 1, n= 73), MetS (group 2, $n=65$ ), and DM (group 3, $n=45$ ). VFA was evaluated by computed tomography $(\mathrm{CT})$ and corrected for body mass index (BMI).

Results: Patients in group 1 had smaller VFAs than patients in groups 2 and $3(3.2 \pm 1.8,4.6 \pm 1.9$, and $4.9 \pm 2.0$ $\mathrm{cm}^{2} /\left[\mathrm{kg} / \mathrm{m}^{2}\right]$, respectively, $\left.P<0.05\right)$ and lower CRP levels $(0.97 \pm 2.31,1.27 \pm 2.57$, and $1.11 \pm 1.35 \mathrm{mg} / \mathrm{dL}$, respectively, $P<0.05)$. VFA increased with the number of criteria met for MetS. After adjusting for age, body weight, and sex, CRP and albumin levels functioned as independent positive predictors of VFA; on other hand, the use of reninangiotensin system blockers was inversely correlated with VFA in PD patients without DM. In the survival analysis, DM patients (group 3) had the poorest survival among the 3 groups, but no significant differences were found between groups 1 and 2 .

Conclusion: This study showed that VFA and MetS are associated with CRP levels but cannot predict survival in PD patients without DM. The complex relationship of nutritional parameters to VFA and MetS may explain these results. The type of antihypertensive medication used was also associated with the VFA. The mechanisms behind these findings warrant further investigation.

Keywords: Abdominal fat, Atherogenic indices, Beta blocker, Renin-angiotensin system blocker, Computed tomography, Metabolic syndrome, Peritoneal dialysis

\footnotetext{
* Correspondence: uynewsh@gmail.com; kyhung@ntu.edu.tw

${ }^{7}$ Department of Internal Medicine, Buddhist Tzu Chi General Hospital,

Taipei Branch, New Taipei City, Taiwan

${ }^{1}$ Department of Internal Medicine, National Taiwan University College of

Medicine and Hospital, No. 7, Chung-Shan South Road, Taipei 100, Taiwan

Full list of author information is available at the end of the article
} 


\section{Introduction}

Visceral fat area (VFA) is known to be correlated strongly with metabolic syndrome (MetS). In the general population, increased VFA is an independent risk factor for atherosclerosis [1-4]. Because VFA plays a key role in the development of diabetogenic, atherogenic, prothrombotic, and proinflammatory MetS, VFA reduction has been proposed as a strategy to prevent atherosclerosis in MetS patients [5].

In patients requiring dialysis, disturbances in lipid and carbohydrate metabolism, which are common, have been associated with VFA and may develop into MetS. In patients with chronic kidney disease (CKD), a higher body mass index (BMI) has been reported to be inversely correlated with mortality; however, other studies have suggested that body composition is a more accurate predictor of survival than BMI $[6,7]$.

VFA can be evaluated accurately by computed tomography $(\mathrm{CT})$ at the level of the umbilicus. In hemodialysis (HD) patients, VFA was found to be the best predictor of atherogenic index (AI), triglyceride (TG) level, and degree of atherosclerosis after correcting for BMI [8,9]. Inflammation

Table 1 Clinical characteristics and biochemical parameters among peritoneal dialysis(PD) patients without metabolic syndrome (MetS, group 1), PD patients with MetS (group 2), and diabetes mellitus (DM) patients (group 3)

\begin{tabular}{|c|c|c|c|c|c|c|c|c|c|}
\hline & & 1 & & & 2 & & & 3 & \\
\hline & & etS, & & & $\mathrm{S}, \mathrm{n}=$ & & & $1, n=$ & \\
\hline & Mean & \pm & SD & Mean & \pm & SD & Mean & \pm & SD \\
\hline Women & & 39 & & & 40 & & & 19 & \\
\hline CAD & & 6 & & & 8 & & & $17^{*}$ & \\
\hline Hypertension & & 55 & & & $58^{*}$ & & & 40 & \\
\hline RAS blocker & & 37 & & & 34 & & & 19 & \\
\hline Beta blocker & & 35 & & & $49^{*}$ & & & 28 & \\
\hline Statin & & 17 & & & 24 & & & 18 & \\
\hline Age & 51 & \pm & 14 & 53 & \pm & 12 & 58 & \pm & $11^{*}$ \\
\hline PD vintage (months) & 46 & \pm & 45 & 49 & \pm & 36 & 22 & \pm & $19^{*}$ \\
\hline Body weight (kg) & 57 & \pm & 9 & 61 & \pm & $12^{*}$ & 68 & \pm & $13^{*}$ \\
\hline Renal KTN & 0.22 & \pm & 0.33 & 0.17 & \pm & 0.32 & 0.18 & \pm & 0.23 \\
\hline Peritoneal KTN & 1.87 & \pm & 0.41 & 1.92 & \pm & 0.35 & 1.79 & \pm & 0.31 \\
\hline 4-h D/P Cre & 0.67 & \pm & 0.10 & 0.64 & \pm & $0.09^{*}$ & 0.68 & \pm & 0.11 \\
\hline D4/D0 Glu & 0.37 & \pm & 0.07 & 0.40 & \pm & $0.06^{*}$ & 0.39 & \pm & 0.07 \\
\hline Glucose exposure (kg/year) & 53 & \pm & 19 & 56 & \pm & 20 & 58 & \pm & 22 \\
\hline Dialysate glucose load (g/dL) & 1.86 & \pm & 0.33 & 1.87 & \pm & 0.31 & 2.11 & \pm & 0.31 \\
\hline nPCR (gm/[kg/day]) & 1.02 & \pm & 0.19 & 0.93 & \pm & $0.16^{*}$ & 0.90 & \pm & $0.21^{*}$ \\
\hline Albumin (g/mdL) & 3.9 & \pm & 0.3 & 4.1 & \pm & $0.3^{*}$ & 4.0 & \pm & 0.4 \\
\hline Hemoglobin (g/dL) & 10.2 & \pm & 1.2 & 10.0 & \pm & 1.6 & 10.2 & \pm & 1.0 \\
\hline Creatinine $(\mathrm{mg} / \mathrm{dL})$ & 11.5 & \pm & 2.9 & 11.2 & \pm & 2.7 & 10.9 & \pm & 2.6 \\
\hline Glucose (mg/dL) & 92 & \pm & 11 & 106 & \pm & $24^{*}$ & 139 & \pm & $52^{*}$ \\
\hline Cholesterol (mg/dL) & 197 & \pm & 44 & 202 & \pm & 48 & 186 & \pm & 41 \\
\hline Triglyceride (mg/dL) & 116 & \pm & 54 & 273 & \pm & $208^{*}$ & 203 & \pm & $133^{*}$ \\
\hline $\mathrm{HDL}(\mathrm{mg} / \mathrm{dL})$ & 48 & \pm & 15 & 37 & \pm & $7^{*}$ & 36 & \pm & $12^{*}$ \\
\hline $\mathrm{LDL}(\mathrm{mg} / \mathrm{dL})$ & 100 & \pm & 39 & 87 & \pm & 42 & 85 & \pm & $37^{*}$ \\
\hline Al1 & 0.36 & \pm & 0.25 & 0.80 & \pm & $0.26^{*}$ & 0.67 & \pm & $0.35^{*}$ \\
\hline $\mathrm{Al} 2$ & 3.31 & \pm & 1.31 & 4.58 & \pm & $1.31^{*}$ & 4.42 & \pm & $1.58^{*}$ \\
\hline CRP $(\mathrm{mg} / \mathrm{dL})$ & 0.97 & \pm & 2.31 & 1.27 & \pm & $2.57^{\mathrm{a}}$ & 1.11 & \pm & $1.35^{\mathrm{a}}$ \\
\hline Cardiothoracic ratio (\%) & 48 & \pm & 7 & 50 & \pm & 7 & 53 & \pm & 6 \\
\hline $\mathrm{SFA}\left(\mathrm{cm}^{2} /\left[\mathrm{kg} / \mathrm{m}^{2}\right]\right)$ & 4.8 & \pm & 2.4 & 6.1 & \pm & $2.0^{*}$ & 7.3 & \pm & $2.8^{*}$ \\
\hline TFA $\left(\mathrm{cm}^{2} /\left[\mathrm{kg} / \mathrm{m}^{2}\right]\right)$ & 8.0 & \pm & 3.6 & 10.7 & \pm & $3.4^{*}$ & 12.1 & \pm & $3.8^{*}$ \\
\hline VFA $\left(\mathrm{cm}^{2} /\left[\mathrm{kg} / \mathrm{m}^{2}\right]\right)$ & 3.2 & \pm & 1.8 & 4.6 & \pm & $1.9^{*}$ & 4.9 & \pm & $2.0^{*}$ \\
\hline
\end{tabular}

${ }^{*} P<0.05$ for comparison to group 1 patients by the student $t$-test.

${ }^{a} P<0.05$ for comparison to group 1 patients by the non-parametric $t$-test.

$S D$ standard deviation, $A / 1$ log (TG/HDL); $A / 2$ non-HDL cholesterol/HDL, BMI Body mass index, CAD coronary artery disease, CRP C-reactive protein, HDL high density lipoprotein, $L D L$ low density lipoprotein, RAS renin-angiotensin system, SFA subcutaneous fat area, TFA total fat area, VFA visceral fat area. 

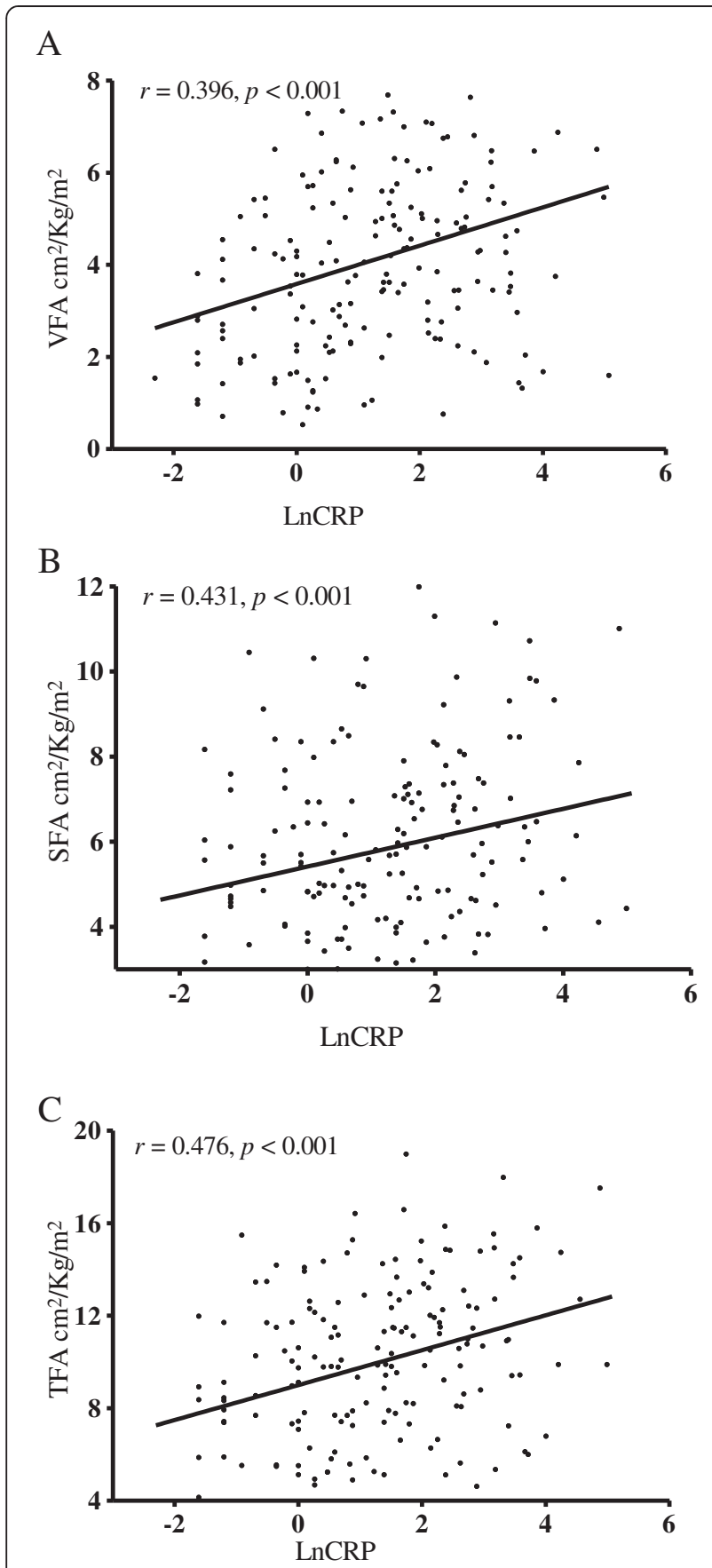

Figure 1 The relationship between fat area and CRP was analyzed with Pearson correlation. Different components of abdominal fat area were all positively correlated with CRP.

is another important component of MetS; in HD [10] and peritoneal dialysis (PD) patients [11], VFA has been found to be associated with increased levels of inflammatory markers such as C-reactive protein (CRP).

An increased glucose concentration in peritoneal fluid results in increased carbohydrate absorption by the peritoneal membrane. The average concentration of glucose in the peritoneal fluid of a PD patient predicts survival and is associated with higher serum glucose levels [12-14]. In one study, approximately one-third of PD patients without DM exhibited insulin resistance [15]; other studies have shown a high incidence $(\geq 50 \%)$ of MetS in this population [16,17]. In PD patients, both insulin resistance and high glucose absorption lead to increased VFA, and thus are associated with higher adipokine concentrations than those in individuals with normal renal function [18]. Therefore, the significant contribution of VFA to increased BMI and the association of VFA with CRP make the elucidation of its role in PD patient outcomes complex.

Some drugs may affect glucose and lipid metabolism and, therefore, influence abdominal fat. Renin-angiotensin system (RAS) blockers have been reported to reduce VFA and decrease vascular inflammation [19,20]. Traditional beta blockers can reduce insulin sensitivity and increase the risk of DM and dyslipidemia [21]. Statins are also used to manage dyslipidemia and insulin resistance [22]. PD patients commonly use these medications to treat hypertension, heart disease, or dyslipidemia; however, the effects of these medications on VFA have never been studied.

This study aimed at clarifying the associations among VFA, MetS, inflammation, and medication use and their corresponding effects on PD patient outcomes. We found that medication use, inflammation, and MetS were all associated with VFA in the PD population.

\section{Materials and methods Study design}

In February 2009, patients aged $>20$ years who had undergone maintenance PD for more than 3 months were enrolled. Pregnant women and patients who had undergone CT scanning during the previous 6 months were excluded. After providing informed consent, each patient underwent a non-contrast abdominal CT, and a blood sample was obtained to measure biomarkers. Patients fasted for 6 hours prior to phlebotomy. The blood samples were immediately centrifuged at 3,000 rpm and $4^{\circ} \mathrm{C}$, and the resulting plasma samples were frozen at $-80^{\circ} \mathrm{C}$ until analysis.

\section{Ethical considerations}

This study was approved by the ethics committee of this hospital in documents NTUH-REC No. 200808062R and NTUH-REC No. 201104032RC. As stated, patients provided written informed consent before they entered the study.

\section{Assessment of abdominal fat by computed tomography}

The imaging of each subject was performed on a 64-MDCT scanner (LightSpeed VCT; GE Healthcare, Milwaukee, WI), and the umbilicus cut was analyzed for VFA [23]. Image 
Table 2 Correlations between abdominal fat and clinical parameters in non-DM $(n=138)$ and DM $(n=45)$ PD patients

\begin{tabular}{|c|c|c|c|c|c|c|c|c|c|c|}
\hline \multirow[b]{3}{*}{ Sex } & \multicolumn{6}{|c|}{ Non-DM, $n=138$} & \multicolumn{4}{|c|}{$\mathrm{DM}, \mathrm{n}=45$} \\
\hline & \multicolumn{2}{|l|}{ VFA } & \multicolumn{2}{|c|}{ SFA } & \multicolumn{2}{|c|}{ TFA } & VFA & \multicolumn{2}{|l|}{ SFA } & \multirow[t]{2}{*}{ TFA } \\
\hline & -0.04 & & 0.31 & * & 0.17 & * & 0.02 & 0.23 & & \\
\hline CAD & 0.06 & & 0.01 & & 0.04 & & 0.25 & 0.02 & & 0.15 \\
\hline Hypertension & -0.15 & & 0.06 & & -0.04 & & 0.31 & 0.06 & & 0.20 \\
\hline RAS blocker & -0.17 & * & -0.17 & * & -0.20 & ${ }^{*}$ & -0.06 & -0.13 & & -0.13 \\
\hline Beta blocker & 0.15 & * & 0.27 & * & 0.24 & * & 0.08 & -0.12 & & -0.04 \\
\hline Statin & 0.10 & & 0.14 & & 0.14 & & -0.08 & -0.12 & & -0.13 \\
\hline Age & 0.40 & * & 0.35 & * & 0.43 & * & 0.50 & 0.06 & & 0.31 \\
\hline PD vintage & 0.00 & & -0.09 & & -0.06 & & -0.20 & -0.04 & & -0.13 \\
\hline Body weight & 0.38 & * & 0.15 & & 0.29 & * & -0.08 & 0.37 & * & 0.23 \\
\hline BMl & 0.44 & * & 0.41 & * & 0.49 & ${ }^{*}$ & 0.02 & 0.54 & * & 0.41 \\
\hline Renal KTN & 0.07 & & 0.15 & & 0.13 & & 0.14 & 0.05 & & 0.10 \\
\hline Peritoneal KTN & -0.18 & * & -0.14 & & -0.18 & * & -0.12 & 0.10 & & 0.02 \\
\hline $4 \mathrm{hr}$ D/P Cre & -0.05 & & -0.04 & & -0.05 & & -0.02 & 0.19 & & 0.13 \\
\hline D4/D0 Glu & 0.15 & & 0.10 & & 0.14 & & -0.03 & -0.15 & & -0.13 \\
\hline Glucose exposure & -0.07 & & -0.07 & & -0.08 & & 0.01 & 0.22 & & 0.16 \\
\hline Dialysate glucose load & -0.02 & & 0.02 & & 0.00 & & 0.09 & -0.07 & & 0.00 \\
\hline $\mathrm{nPCR}$ & -0.23 & * & -0.27 & * & -0.28 & * & -0.25 & -0.21 & & -0.28 \\
\hline Albumin & 0.22 & * & 0.06 & & 0.15 & & 0.09 & -0.03 & & 0.02 \\
\hline Hemoglobin & 0.05 & & 0.03 & & 0.05 & & 0.01 & -0.37 & * & -0.26 \\
\hline Creatinine & -0.13 & & -0.29 & * & -0.25 & * & -0.35 & -0.20 & & -0.32 \\
\hline Glucose & 0.17 & * & 0.10 & & 0.15 & & 0.11 & 0.29 & * & 0.27 \\
\hline Cholesterol & 0.16 & & 0.24 & * & 0.23 & * & 0.24 & 0.22 & & 0.28 \\
\hline Triglyceride & 0.27 & * & 0.12 & & 0.22 & * & 0.54 & 0.34 & * & 0.53 \\
\hline$\overline{\mathrm{HDL}}$ & -0.27 & * & 0.01 & & -0.14 & & -0.26 & -0.22 & & -0.30 \\
\hline LDL & -0.02 & & 0.06 & & 0.03 & & -0.11 & 0.09 & & 0.01 \\
\hline Al1 & 0.42 & * & 0.19 & * & 0.34 & * & 0.55 & 0.41 & * & 0.59 \\
\hline $\mathrm{Al} 2$ & 0.39 & ${ }^{*}$ & 0.18 & * & 0.32 & * & 0.45 & 0.31 & * & 0.46 \\
\hline LnCRP & 0.36 & 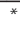 & 0.16 & & 0.29 & ${ }^{*}$ & 0.02 & 0.19 & & 0.15 \\
\hline Cardiothoracic ratio (\%) & 0.25 & * & 0.32 & * & 0.33 & * & 0.32 & 0.23 & & 0.33 \\
\hline
\end{tabular}

Abdominal fat was categorized as visceral fat area (VFA), subcutaneous fat area (SFA), and total fat area (TFA), and all were corrected for body mass index (BMI). ${ }^{*} P<0.05$ with Pearson's correlation.

analysis software (ImageJ, version 1.45; National Institutes of Health, Bethesda, MD) was used at an attenuation range of -50 to -250 Hounsfield units to quantify abdominal adipose tissue areas in $\mathrm{cm}^{2}$. The subcutaneous fat area (SFA) was clearly visible and defined as the extraperitoneal fat between skin and muscle. Intra-abdominal tissue at the same density as SFA was defined as VFA. The total fat area (TFA) was the sum of SFA and VFA. The images were reviewed by radiologists who were blinded to the clinical characteristics of the PD patients. The 3 indicators of fat area were corrected for BMI $\left(\mathrm{cm}^{2} /\left[\mathrm{kg} / \mathrm{m}^{2}\right]\right)$ because fat area relates to body mass [8].

\section{Clinical characteristics and follow-up}

Clinical and dialysis data of PD patients were recorded and included medication (RAS blockers, beta blockers, or statin), results of PD-associated peritoneal equilibration test (PET), dialysis $\mathrm{Kt} / \mathrm{V}$, residual renal function, and normalized protein catabolic rate (nPCR). Routine biochemical studies were also recorded and included urea nitrogen, creatinine, albumin, hemoglobin, CRP, lipid profile, total cholesterol (CHO), triglyceride (TG), high-density lipoprotein (HDL), and lowdensity lipoprotein (LDL).

Two atherogenic indices (AIs) were derived from the lipid profile, according to the following equations: $\mathrm{AI} 1=$ $\log (\mathrm{TG} / \mathrm{HDL})$ [24] and AI2 = non-HDL cholesterol/ $\mathrm{HDL}=(\mathrm{T}-\mathrm{CHO}-\mathrm{HDL}) / \mathrm{HDL}[8]$.

The dialysate prescribed to each PD patient during 2009 was also recorded and used to calculate the glucose load in these patients, as previously described [12-14]. Glucose load 1 represents the average glucose concentration in the total PD fluid, including extraneal and nutrineal. Glucose load 2 
Table 3 Independent determinants of abdominal fat area by multiple linear regression analysis and adjusted for age and sex in non-DM (A) and DM (B) PD patients

A.

\begin{tabular}{|c|c|c|c|c|c|c|c|c|c|c|c|c|}
\hline & & VFA & & & & SFA & & & & $\mathrm{FA}$ & & \\
\hline & B & \pm & SE & $P$ & B & \pm & SE & $P$ & B & \pm & SE & $P$ \\
\hline Constant & -7.58 & \pm & 1.89 & $<0.001$ & -6.86 & \pm & 1.77 & $<0.001$ & -13.34 & \pm & 2.86 & $<0.001$ \\
\hline Woman & 0.51 & \pm & 0.33 & 0.13 & 2.64 & \pm & 0.40 & $<0.001$ & 2.81 & \pm & 0.62 & $<0.001$ \\
\hline Age (per 10 years) & 0.40 & \pm & 0.10 & $<0.001$ & 0.43 & \pm & 0.12 & $<0.001$ & 0.76 & \pm & 0.19 & $<0.001$ \\
\hline BW (per 10 kg) & 0.95 & \pm & 0.15 & $<0.001$ & 1.26 & \pm & 0.18 & $<0.001$ & 2.20 & \pm & 0.27 & $<0.001$ \\
\hline Creatinine & -0.17 & \pm & 0.05 & $<0.01$ & -0.19 & \pm & 0.06 & $<0.01$ & -0.37 & \pm & 0.09 & $<0.001$ \\
\hline Albumin & 0.95 & \pm & 0.37 & $<0.05$ & & & & & & & & \\
\hline $\mathrm{Al} 2$ & 0.30 & \pm & 0.09 & $<0.001$ & & & & & & & & \\
\hline LnCRP & 0.27 & \pm & 0.08 & $<0.001$ & & & & & 0.43 & \pm & 0.14 & $<0.01$ \\
\hline RAS blocker & -0.62 & \pm & 0.25 & $<0.05$ & & & & & -0.92 & \pm & 0.47 & 0.05 \\
\hline Beta blocker & & & & & 1.03 & \pm & 0.30 & $<0.001$ & 1.35 & \pm & 0.45 & $<0.01$ \\
\hline Cholesterol (per 10 mg/dL) & & & & & & & & & 0.11 & \pm & 0.05 & $<0.05$ \\
\hline D4/D0 Glu (per 0.1) & & & & & & & & & 0.67 & \pm & 0.33 & $<0.05$ \\
\hline $\mathrm{R}^{2}$ & & 0.559 & & & & 0.476 & & & & 600 & & \\
\hline
\end{tabular}

B.

\begin{tabular}{|c|c|c|c|c|c|c|c|c|c|c|c|c|}
\hline & \multicolumn{3}{|c|}{ VFA } & \multicolumn{4}{|c|}{ SFA } & \multicolumn{5}{|c|}{ TFA } \\
\hline & B & \pm & SE & $P$ & B & \pm & SE & $\mathbf{P}$ & B & \pm & SE & $\mathbf{P}$ \\
\hline Constant & -0.29 & \pm & 1.30 & 0.83 & -8.10 & \pm & 3.56 & $<0.05$ & -7.60 & \pm & 4.78 & 0.12 \\
\hline Woman & -0.26 & \pm & 0.47 & 0.59 & 2.75 & \pm & 0.73 & $<0.001$ & 2.57 & \pm & 0.97 & $<0.05$ \\
\hline Age (per 10 years) & 0.71 & \pm & 0.21 & $<0.01$ & 0.21 & \pm & 0.33 & 0.53 & 0.80 & \pm & 0.44 & 0.08 \\
\hline Cholesterol (per $10 \mathrm{mg} / \mathrm{dL}$ ) & 0.07 & \pm & 0.02 & $<0.001$ & & & & & & & & \\
\hline BW (per 10 kg) & & & & & 1.28 & \pm & 0.31 & $<0.001$ & 1.20 & \pm & 0.41 & $<0.01$ \\
\hline Al1 & & & & & 2.38 & \pm & 1.02 & $<0.05$ & 4.93 & \pm & 1.37 & $<0.001$ \\
\hline$\overline{R^{2}}$ & 0.445 & & & & 0.464 & & & & 0.491 & & & \\
\hline
\end{tabular}

represents the average glucose concentration of the glucosecontaining dialysate only.

After they had undergone an abdominal CT, patients were monitored for the following events: hospitalization, peritonitis, technique failure, cardiovascular disease (CVD), and mortality. Cardiac disease, cerebrovascular disease, and severe ischemic events were categorized as CVD. Patients who received transplants were censored in technique survival and mortality. Transplant admissions were not considered episodes of hospitalization. Follow-up was continued until February 2012.

\section{Metabolic syndrome}

MetS was diagnosed according to the definition in the National Cholesterol Education Program Adult Treatment Panel III [25], which requires fulfillment of at least 3 of the following criteria: abnormal waist circumference, TG $>150 \mathrm{mg} / \mathrm{dL}, \mathrm{HDL}<40 \mathrm{mg} / \mathrm{dL}$ in men or $<50 \mathrm{mg} / \mathrm{dL}$ in women, blood pressure $>130 /$ $85 \mathrm{mmHg}$ or active treatment with antihypertensive agents, and fasting blood glucose $>100 \mathrm{mg} / \mathrm{dL}$. Because waist measurements are inaccurate in PD patients, we substituted BMI $>25 \mathrm{~kg} / \mathrm{m}^{2}$ for this value, as has been done in other studies [17].

\section{Statistical analysis}

All continuous variables are reported as mean \pm SD (with 95\% confidence intervals as appropriate), and all categorical variables are reported as frequencies or percentages. Comparison between groups was done using the student $t$-test, non-parametric test, or oneway analysis of variance (ANOVA). The differences in frequency were tested by $\chi^{2}$ analysis. The relationships between variables were tested by Pearson's correlation. The independent determinants of a variable were determined by multiple linear regression analysis. The adjusted variables were stated for each analysis. Peritonitis incidence in PD patients was compared by Poisson analysis. Kaplan-Meier survival analysis was used to compare survival between groups. $P$ values < 0.05 were considered significant. Statistical analyses 


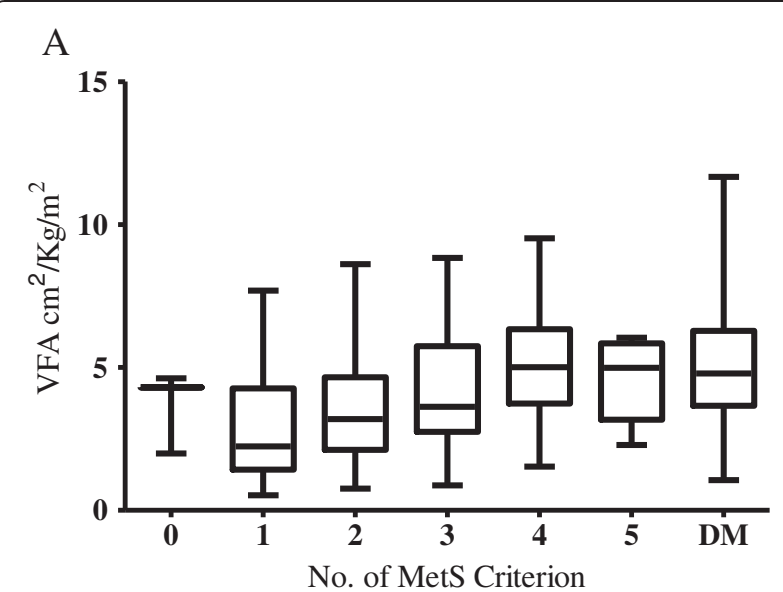

B
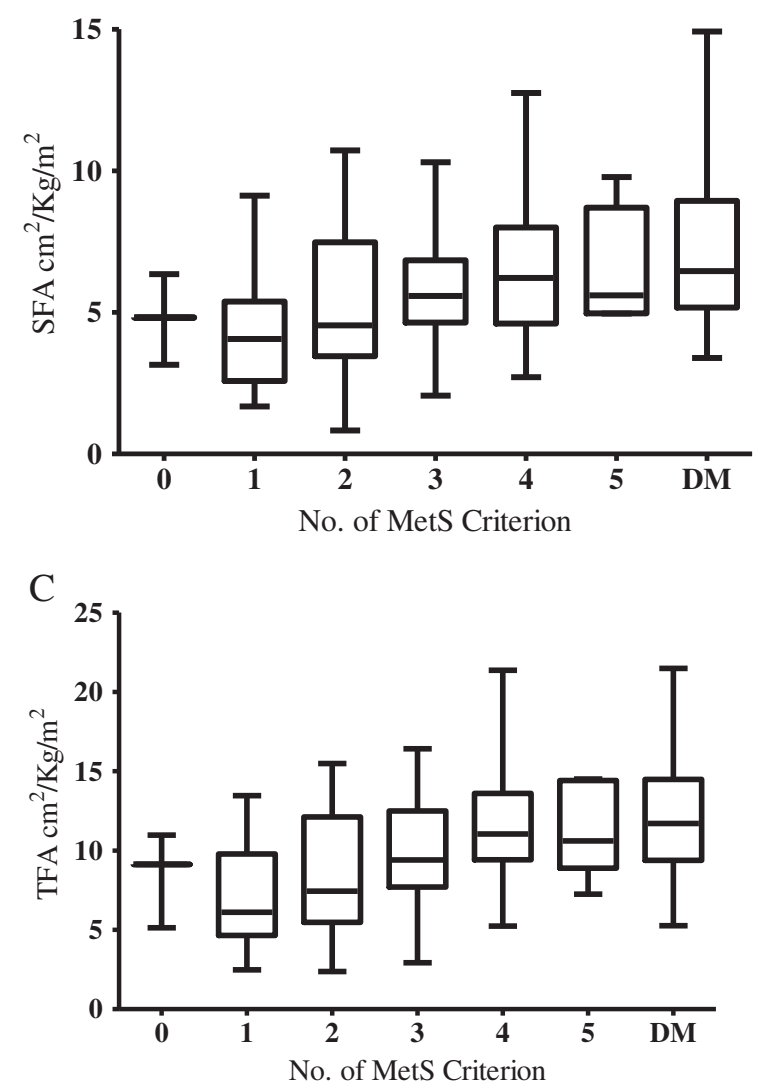

Figure $\mathbf{2}$ The fat area increased as the increment of number of mets criterion in each component of fat area (A) VFA, (B) SFA, and (C) TFA ( $p<0.001$ with ANOVA). The DM patients was categorized as another group in the last bar.

were conducted using SPSS 13.0 for Windows (SPSS Inc., IL USA).

\section{Results}

Clinical and PD-associated parameters among patients A total of 183 PD patients were enrolled in this study. There were 146 patients without DM who were further classified as non-MetS ( $\mathrm{n}=73$, group 1) or MetS $(\mathrm{n}=65$, group 2). The remaining patients were classified as DM $(\mathrm{n}=45$, group 3$)$, including 5 patients met the criteria for DM after they had begun PD. Besides differing in the criteria met for MetS, patients in group 2 also had higher levels of albumin and CRP, higher AI1 and AI2, more abdominal fat indicators, and lower levels of $\mathrm{D} / \mathrm{P}$ creatinine and nPCR than patients in group 1 (Table 1). DM patients (group 3) had more significant histories of coronary artery disease (CAD), older ages, higher BMIs, higher glucose loads, increased AI1 and AI2, and more abdominal fat than patients in group 1 . The PD duration in the DM patients was the shortest and the nPCR was the lowest of the 3 groups. These results indicate that MetS patients are similar to DM patients in that they have higher AIs and CRP levels, and more abdominal fat, but MetS patients have less significant CAD histories.

\section{Predictors for VFA, SFA, and TFA}

Because DM significantly influences lipid and carbohydrate metabolism and fat distribution, we analyzed the correlation between VFA and other variables in the groups. Because CRP was not evenly distributed, we logtransformed the CRP data for analysis. We applied the Pearson's correlation to define the relationship between CRP levels and fat components. In non-DM patients, CRP levels had a significant positive correlation with VFA $(\mathrm{r}=0.396, P<0.001$; Figure 1A), SFA $(\mathrm{r}=0.431, P<$ 0.001; Figure 1B), and TFA $(\mathrm{r}=0.476, \quad P<0.001$; Figure $1 \mathrm{C}$ ). Otherwise, fat area correlated proportionally with age, body weight (BW), CHO, TG, AIs, and fasting glucose (Table 2). These abdominal fat parameters were inversely correlated with peritoneal $\mathrm{KT} / \mathrm{V}$, creatinine, and $\mathrm{nPCR}$. The cardiothoracic ratio, which is inversely related to heart function, also had a positive correlation with abdominal fat. The use of RAS blockers had a negative correlation with abdominal fat, whereas the use of beta blockers had a positive one (Table 2). In general, the correlations were similar in DM patients, except that CRP level and type of antihypertensive medication had no correlations with abdominal fat.

In addition, we used multiple linear regression analysis to determine independent predictors, after adjusting for age, sex, and BW. In non-DM patients, creatinine associated negatively with all fat area indicators (Table 3A). CRP levels were associated positively with VFA and TFA. The use of RAS blockers negatively predicted VFA, whereas the use of beta blockers associated positively with SFA. Both types of medications correlated with TFA. In DM patients, the fat area was associated only with age, sex, and lipid parameters (Table 3B). 
Table 4 Comparison of glucose exposure, inflammation, and CV comorbidity among non-DM PD patients categorized according to each criterion of metabolic syndrome

\begin{tabular}{|c|c|c|c|c|c|c|c|c|c|c|}
\hline & \multicolumn{2}{|c|}{ Triglyceride } & \multicolumn{2}{|c|}{ High density lipoprotein } & \multicolumn{2}{|c|}{ Hypertension } & \multicolumn{2}{|c|}{ Body mass index } & \multicolumn{2}{|c|}{ Glucose } \\
\hline & - & + & - & + & - & + & - & + & - & + \\
\hline & 69 & 69 & 52 & 86 & 22 & 116 & 113 & 25 & 94 & 44 \\
\hline CRP (mg/dL) & $1.0 \pm 2.6$ & $1.2 \pm 2.3$ & $1.0 \pm 2.4$ & $1.2 \pm 2.5^{*}$ & $2.0 \pm 3.7$ & $1.0 \pm 2.1^{*}$ & $0.9 \pm 2.0$ & $2.0 \pm 3.7^{*}$ & $1.0 \pm 2.1$ & $1.4 \pm 3.0^{*}$ \\
\hline $\operatorname{VFA}\left(\mathrm{cm}^{2} /\left[\mathrm{kg} / \mathrm{m}^{2}\right]\right)$ & $3.1 \pm 1.8$ & $4.6 \pm 1.9^{*}$ & $3.2 \pm 1.8$ & $4.2 \pm 2.0^{*}$ & $4.6 \pm 2.0$ & $3.7 \pm 2.0$ & $3.5 \pm 1.8$ & $5.4 \pm 1.9^{*}$ & $3.6 \pm 2.1$ & $4.4 \pm 1.8^{*}$ \\
\hline Cardiothoracic ratio (\%) & $48 \pm 6$ & $49 \pm 7$ & $48 \pm 6$ & $49 \pm 7$ & $47 \pm 7$ & $49 \pm 7$ & $48 \pm 7$ & $50 \pm 5$ & $48 \pm 7$ & $50 \pm 7$ \\
\hline Albumin (g/dL) & $3.9 \pm 0.3$ & $4.1 \pm 0.3^{*}$ & $3.9 \pm 0.4$ & $4.1 \pm 0.3^{*}$ & $4.0 \pm 0.3$ & $4.0 \pm 0.4$ & $4.0 \pm 0.3$ & $4.1 \pm 0.4$ & $4.0 \pm 0.3$ & $4.1 \pm 0.4$ \\
\hline Renal KTN & $0.21 \pm 0.32$ & $0.19 \pm 0.32$ & $0.30 \pm 0.37$ & $0.14 \pm 0.28^{*}$ & $0.10 \pm 0.16$ & $0.22 \pm 0.34^{*}$ & $0.17 \pm 0.29$ & $0.32 \pm 0.44^{*}$ & $0.20 \pm 0.32$ & $0.21 \pm 0.32$ \\
\hline Glucose load (g/dL) & $1.88 \pm 0.33$ & $1.86 \pm 0.31$ & $1.80 \pm 0.29$ & $1.91 \pm 0.33$ & $1.82 \pm 0.31$ & $1.88 \pm 0.32$ & $1.86 \pm 0.32$ & $1.89 \pm 0.34$ & $1.87 \pm 0.33$ & $1.85 \pm 0.29$ \\
\hline Glucose exposure (kg/year) & $56 \pm 20$ & $53 \pm 19$ & $49 \pm 19$ & $58 \pm 20^{*}$ & $53 \pm 12$ & $55 \pm 21$ & $54 \pm 20$ & $57 \pm 21$ & $55 \pm 20$ & $53 \pm 19$ \\
\hline CAD History & 7 & 7 & 3 & 11 & 3 & 11 & 12 & 2 & 8 & 6 \\
\hline CVD Event & 5 & 5 & 3 & 7 & 1 & 9 & 8 & 2 & 7 & 3 \\
\hline
\end{tabular}

${ }^{*} P<0.05$ compared by student $t$-test, non-parametric $t$-test, or Chi-test.

CAD coronary artery disease, CRP C-reactive protein, CVD cardiovascular disease. 


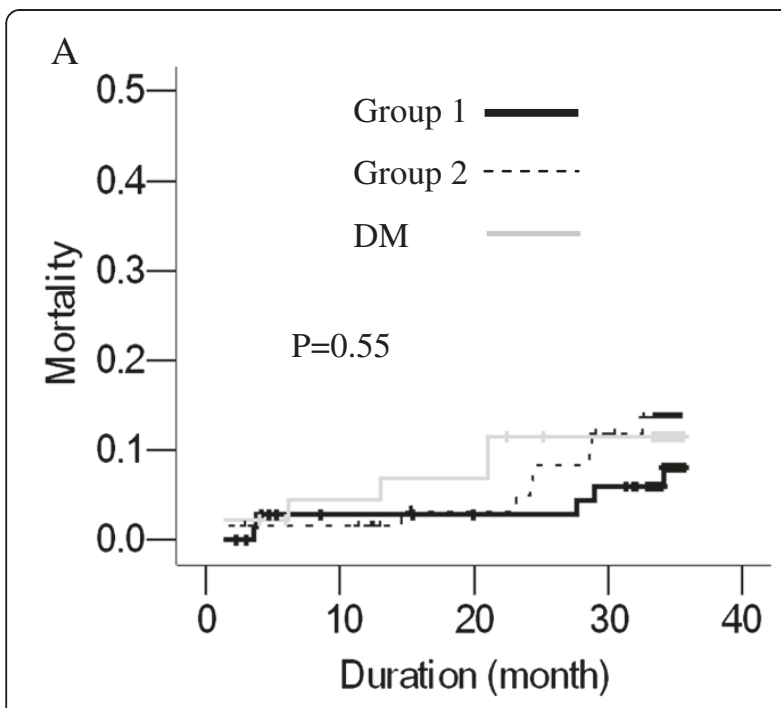

B

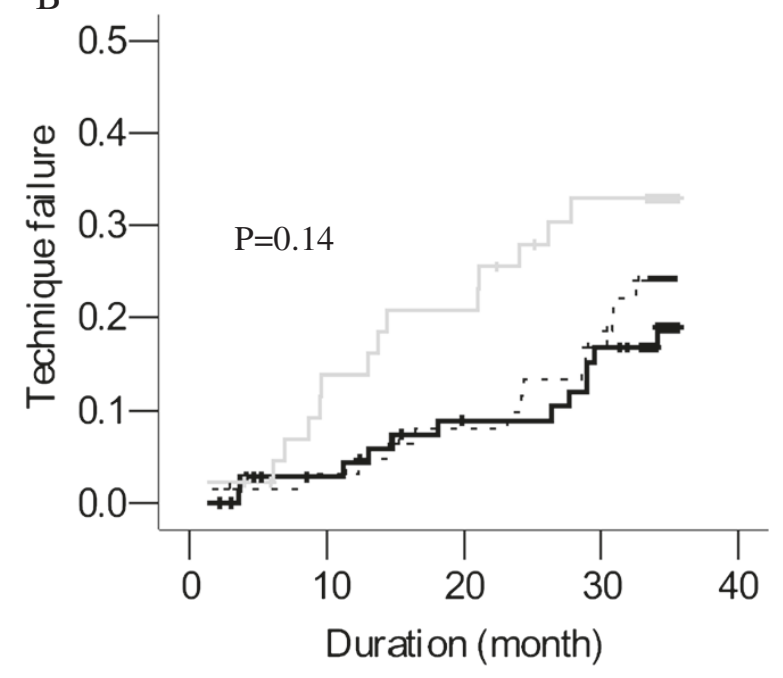

C

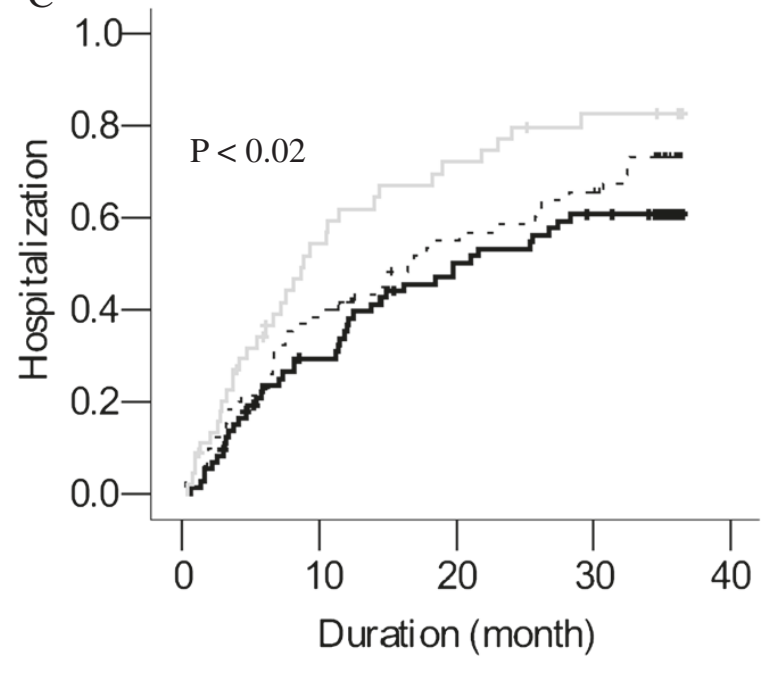

Figure 3 Kaplan-Meir survival analysis was used to compare the duration of time to mortality (A), technique failure (B), and hospitalization (C) among PD patients. All patients were categorized into group 1 non-MetS, group 2 MetS and group 3 DM patients. There was no significant difference among these three groups except that DM patients had shorter time to hospitalization.

\section{Abdominal fat area in MetS}

Since fat area correlated with lipid and glucose metabolism, both of which reflect metabolic dysfunction, we analyzed the fat area in all PD patients without DM and categorized the patients into 6 groups based on the number of criteria they met for MetS. Four patients did not meet any criteria of MetS, 35 fulfilled 1 criterion, 39 fulfilled 2 criteria, 45 fulfilled 3 criteria, 44 fulfilled 4 criteria, and 16 fulfilled all 5 MetS criteria. VFA, SFA, and TFA increased as the number of criteria met increased (Figure 2; $P<0.001$ by ANOVA).

\section{Effects of MetS on PD patient outcomes}

PD patients without DM were analyzed further for each criterion of MetS fulfilled by comparing VFA, CRP, PD dialysate glucose load, annual glucose exposure, CAD history, and CVD comorbidity. CRP levels were higher in patients who fulfilled any single criterion except for hypertension (Table 4). PD glucose load and exposure were similar for each criterion. Finally, none of the criteria for MetS resulted in significant differences in heart size, CAD history, or follow-up CVD events.

The Kaplan-Meier survival analysis was used to compare survival among non-MetS, MetS, and DM patients. Patients with DM had the shortest survival, but MetS did not have an effect on survival outcome (Figure 2).

\section{Discussion}

In this 3-year prospective study, we have demonstrated that VFA, SFA, and TFA increased with the number of MetS criteria met in PD patients. VFA and MetS were also associated with inflammation in PD patients without DM. The use of both RAS blockers and beta blockers was associated with the area of abdominal fat. However, neither MetS nor abdominal fat was associated with technique failure, hospitalization, CVD events, or mortality.

It has been reported that renal failure, especially in association with DM, is associated with the occurrence of CVD events and is a predictor of poor prognosis in patients with acute myocardial infarction [26,27]. MetS is similar to DM in insulin resistance and abnormal glucose and lipid metabolism, and MetS was diagnosed in nearly half of the non-DM PD patients in this study and has been observed in a higher percentage of patients in 
other studies [16,17]. This result is not surprising because one-third of PD patients without DM are glucose intolerant [15]. In addition, PD elevated the daily dialysate glucose load by $1.86 \mathrm{~g} / \mathrm{dL}$ and the annual glucose exposure by $53-56 \mathrm{~kg}$ (Table 1 ), which may affect patients with low HDL levels (Table 4). As in the generalized population [28], PD patients showed an association between MetS and abdominal fat.

Although waist circumference had been reported to be a reliable marker of abdominal adiposity in PD patients [29], waist circumference may be distorted by PD fluid instillation. Fat area measured by the CT scan, as in the present study, would appear to be the more accurate method of measurement. Abdominal fat area increased with the number of criteria fulfilled in PD patients (Figure 2).

MetS in PD patients was also related to inflammation, which is the primary cause of obesity-linked insulin resistance and not only a mere consequence of obesity [30]. Fat accumulation has been linked to inflammation that is characterized by increased CRP levels [31], as shown in the present study. The inflammatory response can be initiated from fat stores [30], or due to uremia, dialysis, or infection [32]. In HD patients, abdominal fat deposition has been linked to inflammation and subsequent increases in mortality risk [33]. In PD patients, visceral fat level was an independent predictor of pulse wave velocity and brachial artery flow-mediated dilatation, and was also one of the risk factors for cardiovascular disease in this population [34]. Our previous study also revealed that larger amounts of adipose tissue were associated with higher serum levels of proinflammatory markers and were, therefore, related to the left ventricular diastolic dysfunction [35].

In our study on PD patients, CRP positively correlated with VFA, SFA, and TFA (Table 2) and remained an independent predictor of fat area after adjusting for sex, age, and BW (Table 3). These results further confirm the relationship between abdominal fat and inflammation in non-DM patients. However, increased abdominal fat and MetS did not influence survival outcomes (Figure 3).

In total, 116 out of 138 (84\%) PD patients with hypertension in our study were prescribed medications to control their BP. However, commonly used antihypertensive agents have different effects on insulin sensitivity, which is associated with abdominal fat. Plasma angiotensin is associated with adiposity [36], and RAS blockers can improve glucose homeostasis [37] and reduce abdominal fat [19,20]. Although we conducted a retrospective analysis of patients' medication use, our PD patients appeared to experience similar RAS blocker-induced effects on VFA. In contrast, traditional beta blockers are known to worsen glucose homeostasis [38]. In the present study, a new generation beta blocker, nebivolol, which may benefit insulin sensitivity
[21], was not used. Beta blockers were positively correlated with SFA. Insulin sensitivity might be influenced by these drugs and the abdominal fat accumulation that follows their use, but to date, their clinical significance in PD patients remains unclear.

This statement raises the question of why CRP-associated abdominal fat does not predict survival in PD patients. Our data may explain this phenomenon. First, BMI had a positive correlation with area of abdominal fat; however, the fat area was adjusted for BMI in this study (Table 2). The inverse association between BMI and mortality in dialysis patients has been established [39]. Second, in addition to CRP and age, albumin was positively correlated with VFA (Table 3). Albumin has been shown to be an important predictor of survival in dialysis patients [40]. With respect to the role of MetS, the analysis of the MetS criteria showed that each criterion did not have a uniform effect in PD patients (Table 4). This study population was composed of fewer obese patients and more hypertensive patients. Obesity has been associated with better clinical outcomes in dialysis patients. Another inverse correlation was also noted between blood pressure and CRP levels, with hypertensive patients having lower CRP levels than normotensive patients. Therefore, these multiple associations complicate the effects of MetS and abdominal fat on PD patient survival.

The present study has certain limitations. First, the followup period is too short to monitor longer-term outcomes such as CVD events and mortality. Second, the small sample size may have influenced the statistical analysis. Since the overall CVD event rate was low in the present study, more patients should be recruited in future studies to determine the effect of abdominal fat area on the CVD event rate. Finally, related to the survival analysis, all the parameters, including VFA, might vary during the study period. As it will not be feasible to measure VFA at regular intervals with CT scans, this will remain a limitation in future studies on survival outcomes.

In conclusion, both abdominal fat area and MetS are associated with inflammation in PD patients. Although the mechanism of the association remains unclear, the use of RAS blockers and beta blockers are associated with abdominal fat area in PD patients without DM. Abdominal fat area and MetS are associated with BMI and nutritional status, and these had an effect opposite to that of inflammation on patient outcomes. These complex associations decrease the capacity of abdominal fat and MetS to predict PD patient outcomes accurately.

\section{Competing interests}

The authors declare that they have no competing interests.

\section{Authors' contributions}

J-WH, Y-CL and K-YH carried out the study design, drafted the manuscript and given final approval of the version to be published. C-YY, H-YW, K-LL, CTS carried out analysis and interpretation of data. C-KW, J-KL, C-KC, H-TC 
carried out acquisition of data and participated in the sequence alignment. All authors read and approved the final manuscript.

\section{Acknowledgement}

This work was supported by a grant from the National Taiwan University Hospital (101- S1924) and the Taipei Branch of the Buddhist Tzu-Chi General Hospital (TCRD-TPE-101-05). The authors thank the staff of the Second Core Lab in the Department of Medical Research of National Taiwan University Hospital for technical support.

\section{Author details}

${ }^{1}$ Department of Internal Medicine, National Taiwan University College of Medicine and Hospital, No. 7, Chung-Shan South Road, Taipei 100, Taiwan. ${ }^{2}$ Medical Imaging, National Taiwan University College of Medicine and Hospital, Taipei, Taiwan. ${ }^{3}$ Integrated Diagnostics and Therapeutics, National Taiwan University College of Medicine and Hospital, Taipei, Taiwan. ${ }^{4}$ Department of Internal Medicine, Far Eastern Memorial Hospital, New Taipei City, Taiwan. ${ }^{5}$ National Taiwan University College of Medicine and Hospital, Yun-Lin Branch, Yun-Lin County, Taiwan. ${ }^{6}$ National Taiwan University College of Medicine and Hospital, Hsin-Chu Branch, Hsin Chu City, Taiwan. ${ }^{7}$ Department of Internal Medicine, Buddhist Tzu Chi General Hospital, Taipei Branch, New Taipei City, Taiwan. ${ }^{8}$ Cardiovascular Center \& Department of Clinical Pathology, Far Eastern Memorial Hospital, New Taipei City, Taiwan.

\section{Received: 13 April 2013 Accepted: 5 June 2013}

Published: 8 June 2013

\section{References}

1. Lear SA, Humphries KH, Kohli S, Frohlich JJ, Birmingham CL, Mancini GB: Visceral adipose tissue, a potential risk factor for carotid atherosclerosis: results of the Multicultural Community Health Assessment Trial (M-CHAT). Stroke 2007, 38(9):2422-2429.

2. Ditomasso D, Carnethon MR, Wright CM, Allison MA: The associations between visceral fat and calcified atherosclerosis are stronger in women than men. Atherosclerosis 2010, 208(2):531-536.

3. Cascella T, Palomba S, De Sio I, Manguso F, Giallauria F, De Simone B, Tafuri D, Lombardi G, Colao A, Orio F: Visceral fat is associated with cardiovascular risk in women with polycystic ovary syndrome. Hum Reprod 2008, 23(1):153-159.

4. Konishi K, Nakano S, Seto H, Tsuda S, Koya D: Carotid atherosclerosis mediated by visceral adiposity and adipocytokines in type 2 diabetic subjects. Diabetes Res Clin Pract 2009, 85(2):171-178.

5. Kishida K, Funahashi T, Matsuzawa Y, Shimomura I: Visceral adiposity as a target for the management of the metabolic syndrome. Ann Med 2012, 44(3):233-241.

6. Mafra D, Guebre-Egziabher F, Fouque D: Body mass index, muscle and fat in chronic kidney disease: questions about survival. Nephrol Dial Transplant 2008, 23(8):2461-2466.

7. Huang JW, Lien YC, Wu HY, Yen CJ, Pan CC, Hung TW, Su CT, Chiang CK Cheng HT, Hung KY: Lean body mass predicts long-term survival in Chinese patients on peritoneal dialysis. PLoS One 2013, 8(1):e54976.

8. Odamaki M, Furuya R, Ohkawa S, Yoneyama T, Nishikino M, Hishida A, Kumagai $\mathrm{H}$ : Altered abdominal fat distribution and its association with the serum lipid profile in non-diabetic haemodialysis patients. Nephrol Dial Transplant 1999, 14(10):2427-2432.

9. Kato A, Ishida J, Endo Y, Takita T, Furuhashi M, Maruyama Y, Odamaki M: Association of abdominal visceral adiposity and thigh sarcopenia with changes of arteriosclerosis in haemodialysis patients. Nephrol Dial Transplant 2011, 26(6):1967-1976.

10. Miyamoto T, Rashid Qureshi A, Heimburger O, Barany P, Carrero K, Sjoberg B, Lindholm B, Stenvinkel P, Carrero JJ: Inverse relationship between the inflammatory marker pentraxin-3, fat body mass, and abdominal obesity in end-stage renal disease. Clin J Am Soc Nephrol 2011, 6(12):2785-2791.

11. Lee MJ, Shin DH, Kim SJ, Oh HJ, Yoo DE, Kim JK, Park JT, Han SH, Kang SW, Choi $\mathrm{KH}$, et al: Visceral fat thickness is associated with carotid atherosclerosis in peritoneal dialysis patients. Obesity (Silver Spring) 2012, 20(6):1301-1307.

12. Wu HY, Hung KY, Huang TM, Hu FC, Peng YS, Huang JW, Lin SL, Chen YM, Chu TS, Tsai TJ, et al: Safety issues of long-term glucose load in patients on peritoneal dialysis--a 7-year cohort study. PLoS One 2012, 7(1):e30337.
13. Wu HY, Hung KY, Huang JW, Chen YM, Tsai TJ, Wu KD: Initial glucose load predicts technique survival in patients on chronic peritoneal dialysis. Am J Nephrol 2008, 28(5):765-771.

14. Wu HY, Hung KY, Hu FC, Chen YM, Chu TS, Huang JW, Wu KD, Tsai TJ: Risk factors for high dialysate glucose use in PD patients--a retrospective 5-year cohort study. Perit Dial Int 2010, 30(4):448-455.

15. Cheng SC, Chu TS, Huang KY, Chen YM, Chang WK, Tsai TJ, Wu KD: Association of hypertriglyceridemia and insulin resistance in uremic patients undergoing CAPD. Perit Dial Int 2001, 21(3):282-289.

16. Chen HY, Kao TW, Huang JW, Chu TS, Wu KD: Correlation of metabolic syndrome with residual renal function, solute transport rate and peritoneal solute clearance in chronic peritoneal dialysis patients. Blood Purif 2008, 26(2):138-144.

17. Li PK, Kwan BC, Ko GT, Chow KM, Leung CB, Szeto CC: Treatment of metabolic syndrome in peritoneal dialysis patients. Perit Dial Int 2009, 29(Suppl 2):S149-S152.

18. Huang JW, Yen CJ, Chiang HW, Hung KY, Tsai TJ, Wu KD: Adiponectin in peritoneal dialysis patients: a comparison with hemodialysis patients and subjects with normal renal function. Am J Kidney Dis 2004, 43(6):1047-1055.

19. Chujo D, Yagi K, Asano A, Muramoto H, Sakai S, Ohnishi A, Shintaku-Kubota M, Mabuchi H, Yamagishi M, Kobayashi J: Telmisartan treatment decreases visceral fat accumulation and improves serum levels of adiponectin and vascular inflammation markers in Japanese hypertensive patients. Hypertens Res 2007, 30(12):1205-1210.

20. Nakagawa N, Yao N, Hirayama T, Ishida M, Ishida H, Wada A, Fujino T, Saijo $Y$, Kikuchi K, Hasebe N: Potential impact of renin-angiotensin system inhibitors and calcium channel blockers on plasma high-molecular -weight adiponectin levels in hemodialysis patients. Hypertens Res 2011, 34(5):592-598.

21. Deedwania P: Hypertension, dyslipidemia, and insulin resistance in patients with diabetes mellitus or the cardiometabolic syndrome: benefits of vasodilating beta-blockers. J Clin Hypertens (Greenwich) 2011, 13(1):52-59.

22. Chan DC, Watts GF: Dyslipidaemia in the metabolic syndrome and type 2 diabetes: pathogenesis, priorities, pharmacotherapies. Expert Opin Pharmacother 2011, 12(1):13-30.

23. Gotoh H, Gohda T, Tanimoto M, Gotoh Y, Horikoshi S, Tomino Y: Contribution of subcutaneous fat accumulation to insulin resistance and atherosclerosis in haemodialysis patients. Nephrol Dial Transplant 2009, 24(11):3474-3480.

24. Dobiasova M, Frohlich J: The plasma parameter log (TG/HDL-C) as an atherogenic index: correlation with lipoprotein particle size and esterification rate in apoB-lipoprotein-depleted plasma (FER(HDL)). Clin Biochem 2001, 34(7):583-588.

25. National Cholesterol Education Program (NCEP) Expert Panel on Detection, Evaluation, and Treatment of High Blood Cholesterol in Adults (Adult Treatment Panel III): Third report of the National Cholesterol Education Program (NCEP) expert panel on detection, evaluation, and treatment of high blood cholesterol in adults (adult treatment panel III) final report. Circulation 2002, 106(25):3143-3421.

26. Lin CC, Chen CC, Kung PT, Li Cl, Yang SY, Liu CS, Lin WY, Lee CC, Li TC, Kardia SL: Joint relationship between renal function and proteinuria on mortality of patients with type 2 diabetes: the taichung diabetes study. Cardiovasc Diabetol 2012, 11:131.

27. Kim CS, Choi JS, Park JW, Bae EH, Ma SK, Jeong MH, Kim YJ, Cho MC, Kim CJ, Kim SW, et al: Concomitant renal insufficiency and diabetes mellitus as prognostic factors for acute myocardial infarction. Cardiovasc Diabetol 2011, 10:95.

28. Bhardwaj S, Misra A, Misra R, Goel K, Bhatt SP, Rastogi K, Vikram NK, Gulati S: High prevalence of abdominal, intra-abdominal and subcutaneous adiposity and clustering of risk factors among urban Asian Indians in North India. PLoS One 2011, 6(9):e24362.

29. Bazanelli AP, Kamimura MA, Manfredi SR, Draibe SA, Cuppari L: Usefulness of waist circumference as a marker of abdominal adiposity in peritoneal dialysis: a cross-sectional and prospective analysis. Nephrol dialy trans off publ European Dialy Trans Assoc European Renal Assoc 2012, 27(2):790-795.

30. Ruan X, Guan Y: Metabolic syndrome and chronic kidney disease. J Diabetes 2009, 1(4):236-245.

31. Guzik TJ, Mangalat D, Korbut R: Adipocytokines - novel link between inflammation and vascular function? J Physiol Pharmacol 2006, 57(4):505-528. 
32. Kaysen GA: The microinflammatory state in uremia: causes and potential consequences. J Am Soc Nephrol 2001, 12(7):1549-1557.

33. Cordeiro AC, Qureshi AR, Stenvinkel P, Heimburger O, Axelsson J, Barany P, Lindholm B, Carrero JJ: Abdominal fat deposition is associated with increased inflammation, protein-energy wasting and worse outcome in patients undergoing haemodialysis. Nephrol Dial Transplant 2010, 25(2):562-568.

34. Lu Q, Cheng LT, Wang T, Wan J, Liao LL, Zeng J, Qin C, Li KJ: Visceral fat, arterial stiffness, and endothelial function in peritoneal dialysis patients. $J$ renal nutri off j Council Renal Nutri Nat Kidney Found 2008, 18(6):495-502.

35. Wu CK, Huang YT, Lin HH, Yang CY, Lien YC, Lee JK, Huang JW, Hung KY: Dissecting the mechanisms of left ventricular diastolic dysfunction and inflammation in peritoneal dialysis patients. PLoS One 2013, 8(5):e62722.

36. Saiki A, Ohira M, Endo K, Koide N, Oyama T, Murano T, Watanabe H, Miyashita Y, Shirai K: Circulating angiotensin II is associated with body fat accumulation and insulin resistance in obese subjects with type 2 diabetes mellitus. Metabolism 2009, 58(5):708-713.

37. McMurray JJ, Holman RR, Haffner SM, Bethel MA, Holzhauer B, Hua TA, Belenkov Y, Boolell M, Buse JB, Buckley BM, et al: Effect of valsartan on the incidence of diabetes and cardiovascular events. N Engl J Med 2010, 362(16):1477-1490.

38. Bakris GL, Fonseca V, Katholi RE, McGill JB, Messerli FH, Phillips RA, Raskin P, Wright JT Jr, Oakes R, Lukas MA, et al: Metabolic effects of carvedilol vs metoprolol in patients with type 2 diabetes mellitus and hypertension: a randomized controlled trial. JAMA 2004, 292(18):2227-2236.

39. Kovesdy CP, Anderson JE, Kalantar-Zadeh K: Paradoxical association between body mass index and mortality in men with CKD not yet on dialysis. Am J Kidney Dis 2007, 49(5):581-591.

40. Perl J, Bargman JM: The importance of residual kidney function for patients on dialysis: a critical review. Am J Kidney Dis 2009, 53(6):1068-1081.

doi:10.1186/1475-2840-12-86

Cite this article as: Huang et al: Metabolic syndrome and abdominal fat are associated with inflammation, but not with clinical outcomes, in peritoneal dialysis patients. Cardiovascular Diabetology 2013 12:86.

\section{Submit your next manuscript to BioMed Central and take full advantage of:}

- Convenient online submission

- Thorough peer review

- No space constraints or color figure charges

- Immediate publication on acceptance

- Inclusion in PubMed, CAS, Scopus and Google Scholar

- Research which is freely available for redistribution 